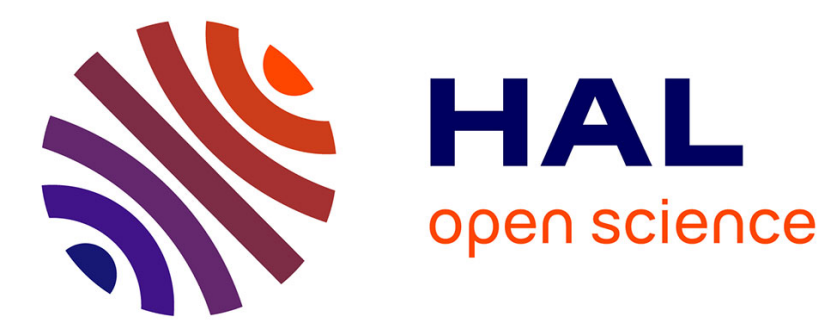

\title{
Micromagnetic analysis of dynamical bubble-like solitons based on the time domain evolution of the topological density
}

Vito Puliafito, Luis Torres, Ozhan Ozatay, Thomas Hauet, Bruno Azzerboni, Giovanni Finocchio

\section{To cite this version:}

Vito Puliafito, Luis Torres, Ozhan Ozatay, Thomas Hauet, Bruno Azzerboni, et al.. Micromagnetic analysis of dynamical bubble-like solitons based on the time domain evolution of the topological density. Journal of Applied Physics, 2014, 115 (17), pp.17D139. 10.1063/1.4867747 . hal-01282861

\section{HAL Id: hal-01282861 https://hal.science/hal-01282861}

Submitted on 13 Jul 2016

HAL is a multi-disciplinary open access archive for the deposit and dissemination of scientific research documents, whether they are published or not. The documents may come from teaching and research institutions in France or abroad, or from public or private research centers.
L'archive ouverte pluridisciplinaire HAL, est destinée au dépôt et à la diffusion de documents scientifiques de niveau recherche, publiés ou non, émanant des établissements d'enseignement et de recherche français ou étrangers, des laboratoires publics ou privés. 


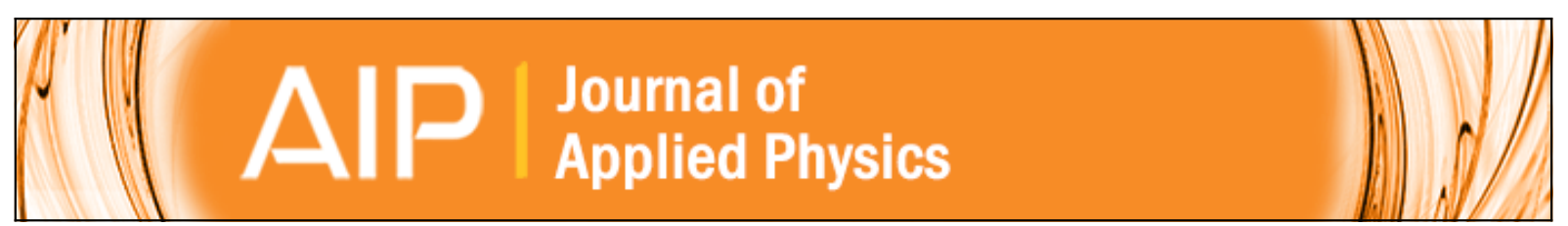

Micromagnetic analysis of dynamical bubble-like solitons based on the time domain evolution of the topological density

Vito Puliafito, Luis Torres, Ozhan Ozatay, Thomas Hauet, Bruno Azzerboni, and Giovanni Finocchio

Citation: Journal of Applied Physics 115, 17D139 (2014); doi: 10.1063/1.4867747

View online: http://dx.doi.org/10.1063/1.4867747

View Table of Contents: http://scitation.aip.org/content/aip/journal/jap/115/17?ver=pdfcov

Published by the AIP Publishing

\section{$\stackrel{A}{A} \mathbb{P} P$ Re-register for Table of Content Alerts}

\section{Create a profile.}




\title{
Micromagnetic analysis of dynamical bubble-like solitons based on the time domain evolution of the topological density
}

\author{
Vito Puliafito, ${ }^{1, a)}$ Luis Torres, ${ }^{2}$ Ozhan Ozatay, ${ }^{3}$ Thomas Hauet, ${ }^{4}$ Bruno Azzerboni, ${ }^{1}$ \\ and Giovanni Finocchio ${ }^{1}$ \\ ${ }^{1}$ Department of Electronic Engineering, Industrial Chemistry and Engineering, University of Messina, 98166 \\ Messina, Italy \\ ${ }^{2}$ Department of Applied Physics, University of Salamanca, 37008 Salamanca, Spain \\ ${ }^{3}$ Department of Physics, Bogazici University, 34342 Bebek/Istanbul, Turkey \\ ${ }^{4}$ Institut Jean Lamour, Université de Lorraine-CNRS UMR 7198, 54506 Nancy, France
}

(Presented 5 November 2013; received 23 September 2013; accepted 5 December 2013; published online 7 March 2014)

\begin{abstract}
Dynamical bubble-like solitons have been recently investigated in nanocontact-based spin-torque oscillators with a perpendicular free layer. Those magnetic configurations can be excited also in different geometries as long as they consist of perpendicular materials. Thus, in this paper, a systematic study of the influence of both external field and high current on that kind of dynamics is performed for a spin-valve point-contact geometry where both free and fixed layers present strong perpendicular anisotropy. The usage of the topological density tool highlights the excitation of complex bubble/antibubble configurations. In particular, at high currents, a deformation of the soliton and its simultaneous shift from the contact area are observed and can be ascribable to the Oersted field. Results provide further detailed information on the excitation of solitons in perpendicular materials for application in spintronics, magnonics, and domain wall logic. (C) 2014 AIP Publishing LLC. [http://dx.doi.org/10.1063/1.4867747]
\end{abstract}

\section{INTRODUCTION}

In the last years, a great attention of the scientific community has been addressed to spin-wave excitations in magnetic nanostructures with non-uniform magnetization distributions. The reason of such an interest lies both in the fundamental physics involved in those phenomena and in the various potential applications that range from high-density storage media to logical integrated circuits, passing through spintronic devices. ${ }^{1-3}$

Among the magnetic materials used for exciting spinwave dynamics, thin ferromagnetic layers with strong perpendicular magnetic anisotropy (PMA) prove great properties and versatility. In particular, in a nanocontact-based spin-torque oscillator, where the free layer (FL) presented PMA, it was possible to observe and study the nucleation of dynamical bubble-like (or droplet) solitons. ${ }^{4,5}$

A magnetic bubble is referred to as a bi-domain state, namely, two concentric up and down domains separated by an in-plane domain wall (DW) ${ }^{6,7}$ The shape of the inner domain can be circular, in the most simple configurations, or irregular. Actually, to characterize the topological complexity of this kind of structures, it is possible to evaluate the topological density $n(x, y)=\left(\partial_{x} \mathbf{m} \times \partial_{y} \mathbf{m}\right) \cdot \mathbf{m}$, which is defined at every point of the film and indicates how the magnetization vector $\mathbf{m}$ varies locally in the plane. Moreover, the integral of $n$ over the film plane, namely, the topological charge $\mathcal{N}^{8}$ always turns out an integer for a magnetic bubble. In detail, the bubble with $\mathcal{N}=1$, corresponding to a topological density $n$ positive at the DW and zero elsewhere, is

${ }^{\text {a)} E l e c t r o n i c ~ m a i l: ~ v p u l i a f i t o @ u n i m e . i t . ~}$ usually simply called a bubble, while the one with $\mathcal{N}=-1$, corresponding to negative $n$ at the DW, is called an antibubble. In some cases, magnetic configurations with $\mathcal{N}=0$ can be excited and correspond to a topological density which is positive in some regions, negative in others. For this reason, we can refer to those configurations as pairs of bubble/antibubble. We underline that the previous definitions refer to static magnetic configurations. On the other hand, when a dynamical bubble-like soliton is excited, the topological density configuration is dynamical as well, and in strong non-linear configurations the topological charge can even change in time.

Here, with this regard, a micromagnetic numerical analysis on a spin valve point-contact geometry made of materials with strong PMA is reported. Such geometry is different from the one in Ref. 4, as free and fixed layers are both perpendicular, and as its non-extended dimensions entail, among other things, diverse numerical approaches and particular kinds of dynamics. ${ }^{2}$ Nonetheless, it allows the excitation of similar bubble-like solitons. In detail, we show the magnetization dynamics excited by the current with both zero and increasing in-plane field, highlighting the time domain evolution of the topological properties. At the critical current, two pairs of bubble/antibubble are nucleated and rotate in a circular trajectory below the nanocontact. As the current increases, a different scenario is achieved where the Oersted field plays the crucial role in breaking the symmetry of the dynamics. In particular, at very high applied current, together with the rotation of the bubble-antibubble pairs, a shift of the soliton from the center of the contact is induced. Finally, we will discuss the influence of the thermal field on the numerical results. 


\section{MICROMAGNETIC FRAMEWORK}

Schematics of the spin-torque oscillator under investigation is shown in the top inset of Fig. 1(a). The spin valve consists of a soft thin ferromagnetic layer and a thick hard layer, usually referred to as FL and pinned layer (PL), respectively, separated by a nonferromagnetic layer. Both FL and PL are composed of perpendicular materials. A current is applied perpendicularly to the structure by means of a metallic circular nano-contact, realized on the top of the FL. The dynamics of the magnetization vector of the FL, $\mathbf{M}=\mathbf{M}(t, \mathbf{r})$, is governed by the Landau-Lifshitz-GilbertSlonczewski (LLGS) equation, ${ }^{9}$

$$
\begin{aligned}
\frac{\partial \mathbf{M}}{\partial t}= & \gamma\left[\mathbf{H}_{\text {eff }} \times \mathbf{M}\right]+\frac{\alpha}{M_{\mathrm{S}}}\left[\mathbf{M} \times \frac{\partial \mathbf{M}}{\partial t}\right] \\
& +f\left(r / R_{\mathrm{c}}\right) \frac{\sigma I}{M_{\mathrm{S}}}[\mathbf{M} \times(\mathbf{M} \times \mathbf{p})] .
\end{aligned}
$$

A detailed description of Eq. (1) can be found elsewhere. ${ }^{10}$ Here, we stress that the effective magnetic field $\mathbf{H}_{\text {eff }}$ includes magnetostatic, exchange, Oersted, Zeeman, magnetostatic coupling, and magneto-crystalline anisotropy contributions. The coefficient $\sigma$ is related to the dimensionless spin polarization efficiency $\varepsilon=a /(1+b \cos \theta)$, where $\theta$ is the angle between the magnetization of the PL and the FL, $a$ and
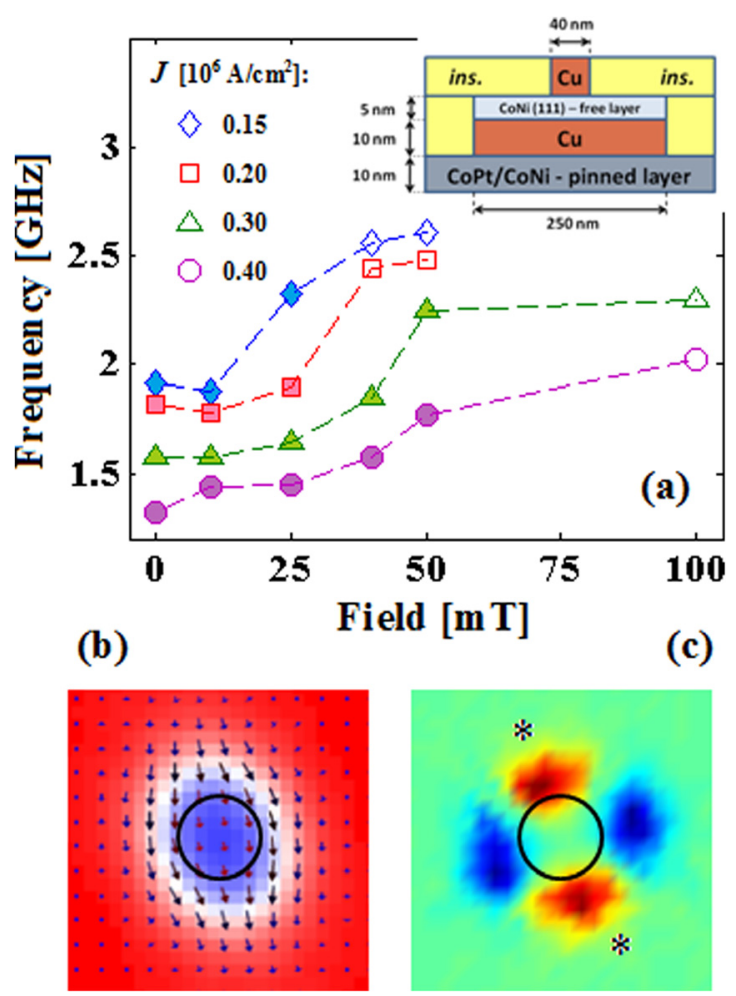

FIG. 1. (a) Output frequency vs. external in-plane field for different values of the applied current. Filled symbols represent frequencies of unstable bubbles. (top-right inset) Longitudinal section of the geometry under investigation. The cross section is circular. (b) Frame from the video of the magnetization configuration and (c) corresponding topological density in a zoomed area $\left(150 \times 150 \mathrm{~nm}^{2}\right.$ around the center) of the FL at zero field and $J=0.15 \times 10^{6}$ $\mathrm{A} / \mathrm{cm}^{2}$. Black continuous circles here and in future plots indicate the contact boundary (Multimedia view) [URL: http://dx.doi.org/10.1063/1.4867747.1] [URL: http://dx.doi.org/10.1063/1.4867747.2]. $b$ have been computed considering the experimental data (the values of the critical currents and asymmetry between them) of a similar structure and their values are 0.54 and 0.6 , respectively. ${ }^{11,12}$ In our approach, moreover, the LLGS equation is numerically solved by using a 3D dynamical code. ${ }^{13}$

Concerning to the detailed parameters used in our framework, PL is $10 \mathrm{~nm}$ thick and made of $\mathrm{CoPt} / \mathrm{CoNi}$, the spacer is 10 -nm-thick $\mathrm{Cu}$, and FL is 5 -nm-thick CoNi. The cross section of the spin-valve is circular with a diameter of $250 \mathrm{~nm}$. The contact is circular as well, centrally positioned on the top of the FL, with a diameter of $40 \mathrm{~nm}$. We used a 2D mesh of discretization cells having sizes $5 \times 5 \times 5 \mathrm{~nm}^{3}$. The magnetic parameters used for the FL simulations are typical experimental values: saturation magnetization is $M_{\mathrm{S}}=650 \times 10^{3} \mathrm{~A} / \mathrm{m}$, exchange constant is $A=1.3 \times 10^{-11}$ $\mathrm{J} / \mathrm{m}$, and perpendicular magnetic anisotropy constant is $K_{\mathrm{u}}=3.3 \times 10^{5} \mathrm{~J} / \mathrm{m}^{3}$. Concerning to the PL, it acts as polarizer for the spins of the current and it is considered fixed in our simulations. Stray field due to its magnetization is calculated only once and introduced in the simulations as additional contribution to the effective field.

\section{RESULTS AND DISCUSSION}

A magnetic bubble can be opportunely excited by simply applying an enough large current to the contact of the spinvalve. The nucleation of the bubble, in fact, occurs also with no external field. The inclusion of an in-plane field, nonetheless, modifies the excited dynamics. With this regard, Fig. 1(a) shows, as obtained by means of numerical simulations, the dependence of the output frequency of the excited dynamics on the external in-plane field strength for different values of the applied current. The first evidence is that the output frequency increases by increasing the field but it decreases by increasing the current. The excited dynamics, after a short nucleation transient, corresponds to a dynamical bi-domain state, represented in the frame of Fig. 1(b) (Multimedia view). In detail, we see an inner area of the FL (blue color online), rotating under the contact area and around its center, where the magnetization points down (more precisely the magnetization precesses with a negative vertical $z$-component), separated from the area where the magnetization points up (red color online) by means of a DW with in-plane magnetization. The main difference between zero field condition and in-plane field inclusion is that in the latter case the inner area gets smaller when the in-plane magnetization of the DW is in the opposite direction with respect to the external field. Fig. 1(c) (Multimedia view) represents the topological density associated to the frame in Fig. 1(b). Once the transient is finished, there appear two areas indicated with stars (red color online) where $n$ assumes positive values and two more areas (blue color online) where $n$ is negative. For this reason, the excitation corresponds to two pairs of bubble/antibubble rotating as the magnetization configuration does. It is also important to stress that the external in-plane field introduces a strong asymmetry in the magnetic configuration. For this reason, the higher is the field the higher is the current needed to excite a stable bubble-like soliton. Concerning to that, in Fig. 1(a), we have filled the symbols for frequencies corresponding to stable 
solitons, leaving empty the symbols corresponding to unstable ones. In this latter case, the soliton first is nucleated and then disappears, continuously, without getting a stable dynamics.

The regular dynamics of the soliton mode drastically changes as the current gets high values. Fig. 2(a) (Multimedia view) shows the magnetic configuration obtained by applying a very high current of $2 \times 10^{6} \mathrm{~A} / \mathrm{cm}^{2}$ at zero field. In this case, we see that the soliton simultaneously rotates around itself and shifts from contact area, losing its symmetry with respect to the contact area and assuming irregular shapes. Such behavior numerically reproduces those particular "spinning" and "breathing" dynamics, observed and described in Ref. 4 for a nanocontact-based spin-torque oscillator. Concerning to the topological density, its basic configuration is the same as the previous case, but it dynamically changes with the nucleation and denucleation of areas with positive or negative $n$ (see Fig. 2(b), Multimedia view). This behavior happens independently of the field, although the concomitant presence of an high current and an high field can induce a complete reversal of the magnetization in the FL from the up state to the down state. Thus, the reason of that irregular behavior at high currents is clearly related to the Oersted field that breaks the symmetry of the effective field. This latter conclusion is also confirmed by simulations performed without Oersted field, where the excited bubble returns regular and the shift disappears.

Finally, we report the results of the inclusion of the thermal field in our micromagnetic framework. Basically, the temperature does not modify qualitatively the excited dynamics but simply introduces the phase noise of the oscillator. With this regard, Fig. 3(a) shows the $z$-component of the magnetization under the contact area with $\mathrm{T}=300 \mathrm{~K}$, a $50 \mathrm{mT}$ in-plane field, and a current of $0.20 \times 10^{6} \mathrm{~A} / \mathrm{cm}^{2}$, whereas Fig. 3(b) (Multimedia view) represents a snapshot of the magnetization configuration. As in the case with no temperature, the excited bubble-like soliton is not stable since it appears and disappears in time. Nevertheless, also in

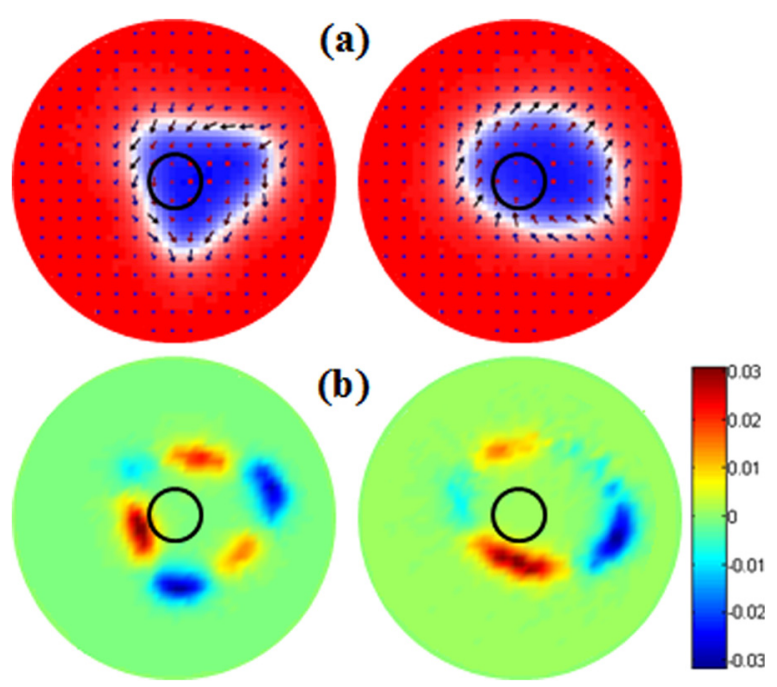

FIG. 2. (a) Frames from the video of the FL magnetic configuration at zero field and $J=2 \times 10^{6} \mathrm{~A} / \mathrm{cm}^{2}$ and (b) corresponding topological density (Multimedia view) [URL: http://dx.doi.org/10.1063/1.4867747.3] [URL: http://dx.doi.org/10.1063/1.4867747.4].
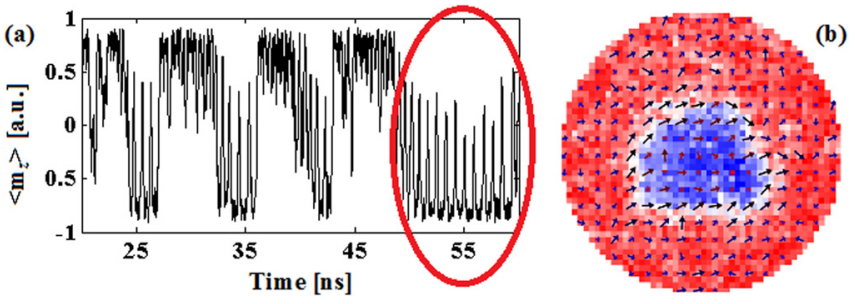

FIG. 3. Results for $\mathrm{T}=300 \mathrm{~K}, H=50 \mathrm{mT}$, and $J=0.20 \times 10^{6} \mathrm{~A} / \mathrm{cm}^{2}$. (a) Average $z$-component of the magnetization under the contact area. In the highlighted interval a bubble-like soliton is excited. (b) Frame from the video of the FL magnetic configuration in the interval between 55 and $58 \mathrm{~ns}$ (Multimedia view) [URL: http://dx.doi.org/ 10.1063/1.4867747.5].

those cases where we had obtained stable excitations of bubble-like solitons, simulations performed with temperature confirm the same evidences.

In summary, we have numerically shown the effect of the external field and of a high current on the bubble-like solitons excited in a point-contact geometry. The external field introduces a shrinkage of the soliton when the magnetization points against the field. The high current, due to the role of the Oersted field, deforms the soliton and shift it from the contact area. Furthermore, we have characterized those solitons by computing the associated topological density. This tool has allowed to highlight the nucleation of bubble/antibubble pairs that rotate and more generally suffer the same behavior as the magnetic configuration. Simulations with the thermal field do not reveal any qualitative change in the excited dynamics.

\section{ACKNOWLEDGMENTS}

This work was supported by Project PRIN2010ECA8P3 from Italian MIUR, Project MAT2011-28532-C03-01 from Spanish government, and Project SA163A12 from Junta de Castilla y Leon.

${ }^{1}$ S. A. Wolf, D. D. Awschalom, R. A. Buhrman, J. M. Daughton, S. von Molnar, M. L. Roukes, A. Y. Chtchelkanova, and D. M. Treger, Science 294, 1488 (2001).

${ }^{2}$ Z. Zeng, G. Finocchio, and H. Jiang, Nanoscale 5, 2219 (2013).

${ }^{3}$ W. Kleemann, J. Appl. Phys. 114, 027013 (2013).

${ }^{4}$ S. M. Mohseni, S. R. Sani, J. Persson, T. N. Anh Nguyen, S. Chung, Ye. Pogoryelov, P. K. Muduli, E. Iacocca, A. Eklund, R. K. Dumas, S. Bonetti, A. Deac, M. A. Hoefer, and J. Åkerman, Science 339, 1295 (2013).

${ }^{5}$ M. A. Hoefer, T. J. Silva, and M. W. Keller, Phys. Rev. B 82, 054432 (2010).

${ }^{6}$ N. Vukadinovic and F. Boust, Phys. Rev. B 84, 224425 (2011).

${ }^{7}$ C. Moutafis, S. Komineas, C. A. F. Vaz, J. A. C. Bland, T. Shima, T. Seki, and K. Takanashi, Phys. Rev. B 76, 104426 (2007).

${ }^{8}$ N. Papanicolaou and T. N. Tomaras, Nucl. Phys. B 360, 425 (1991).

${ }^{9}$ J. Slonczewski, J. Magn. Magn. Mater. 159, L1 (1996).

${ }^{10}$ V. Puliafito, G. Consolo, and B. Azzerboni, IEEE Trans. Magn. 48, 4786 (2012)

${ }^{11}$ O. Ozatay, N. C. Emley, P. M. Braganca, A. G. F. Garcia, G. D. Fuchs, I. N. Krivorotov, R. A. Buhrman, and D. C. Ralph, Appl. Phys. Lett. 88, 202502 (2006).

${ }^{12}$ G. Finocchio, O. Ozatay, L. Torres, R. A. Buhrman, D. C. Ralph, and B. Azzerboni, Phys. Rev. B 78, 174408 (2008).

${ }^{13}$ G. Finocchio, A. Prattella, G. Consolo, E. Martinez, A. Giordano, and B. Azzerboni, J. Appl. Phys. 110, 123913 (2011). 